\title{
Synthesis, Antitumor and Antimicrobial Activities of Some Novel 1- (Substituted)-3-Methyl-1H-Pyrazol-5(4H)-One
}

\author{
R. V. Antre ${ }^{1 *}$, A. Cendilkumar ${ }^{2}$, R. Nagarajan ${ }^{3}$, D. Goli ${ }^{2}$, and R. J. Oswal ${ }^{1}$ \\ ${ }^{1}$ Medicinal Chemistry Research Laboratory, JSPM`S Charak College of Pharmacy and Research, \\ Wagholi, Pune, Maharashtra 412207, India \\ ${ }^{2}$ Medicinal Chemistry Research Laboratory, Acharya and B.M. Reddy College of Pharmacy, \\ Bangalore, Karnataka 560090, India \\ ${ }^{3}$ Organic Chemistry Research Laboratory, School of Chemistry, University of Hyderabad, Central \\ University (P.O.), Hyderabad, Andhrapradesh 500046, India
}

Received 8 February 2011, accepted in final revised form 20 October 2011

\begin{abstract}
Several 1-(substituted)-3-methyl-1H-pyrazol-5(4H)-one as RA1-RA9 were synthesized and compounds were screened for antitumor activity against Ehrlich ascites carcinoma (EAC) cells and antimicrobial activity. Elemental analysis, mass spectral data, ${ }^{1} \mathrm{H}-\mathrm{NMR}$, and IR confirmed the structure of the newly synthesized compounds. Some of the tested compounds showed good antitumor and antimicrobial activity. Compounds RA1, RA4, and RA9 exhibit highest antitumor activity against EAC cells in comparison with 5-flurouracil as standard drug.

Keywords: Ehrlich ascites carcinoma; Pyrazolone; Antitumor; N-substitution; Antimicrobial.

(c) 2012 JSR Publications. ISSN: 2070-0237 (Print); 2070-0245 (Online). All rights reserved. doi:10.3329/jsr.v4i1.7027

J. Sci. Res. 4 (1), 183-192 (2012)
\end{abstract}

\section{Introduction}

Pyrazolone ring system has been consistently rewarded as a promising molecule because of its broad spectrum pharmacological activities like antitubercular [1], antimicrobial [2], anticancer [3,4], antiviral [5], analgesic [6-9], anti-inflammatory [8, 9], antipyretic [9, 10], ulcerogenic [11], lipid peroxidation [11]. Structure activity relationship studies of pyrazolone ring system revealed in various literatures [12-14], suggest position $\mathrm{N}-1, \mathrm{C}-3$, C-4 are very much important for structure activity studies and C-3 should be attached to different heterocyclic rings for better chemotherapeutic activity. Since N-substitutions in pyrazolone exhibit biologically active compounds [15], we were interested in preparing compounds containing them. In view of these observations, we report herein the reaction of 3-methyl-1H-pyrazol-5(4H)-one (1) and 1,2-dibromo ethane in acetonitrile with

\footnotetext{
* Corresponding author: rishiantre@gmail.com
} 
presence of $\mathrm{K}_{2} \mathrm{CO}_{3}$ forms 1-(2-bromoethyl)-3-methyl-1H-pyrazol-5(4H)-one (2). Compound 2 further reacted with substituted amines gives formation of compound RA1RA9. The compound RA1-RA9 screened for their antitumor as well as antimicrobial activities.

\section{Chemistry}

In scheme-1 ethyl acetoacetate was reacted with hydrazine hydrate in presence of ethanol forms 3-methyl- $1 H$-pyrazol-5(4H)-one (1). The treatment of $\mathbf{1}$ with 1,2-dibromo ethane in acetonitrile with presence of $\mathrm{K}_{2} \mathrm{CO}_{3}$ forms 1-(2-bromoethyl)-3-methyl- $1 \mathrm{H}$-pyrazol-5(4H)one 2. This 2 reacts with substituted amines forms RA1-RA9. The ${ }^{1} \mathrm{H}-\mathrm{NMR}$ spectra of 3methyl-1H-pyrazol-5(4H)-one (1) showed broad peak of -NH at $\delta 10.5 \mathrm{ppm}$ two protons and presence of broad peak at $\delta 9.086 \mathrm{ppm}$ due to one proton established that $\mathrm{N}$ substitution occurs in product (2). The ${ }^{1} \mathrm{H}-\mathrm{NMR}$, MS, IR and elemental analysis supported the structure of title compounds.

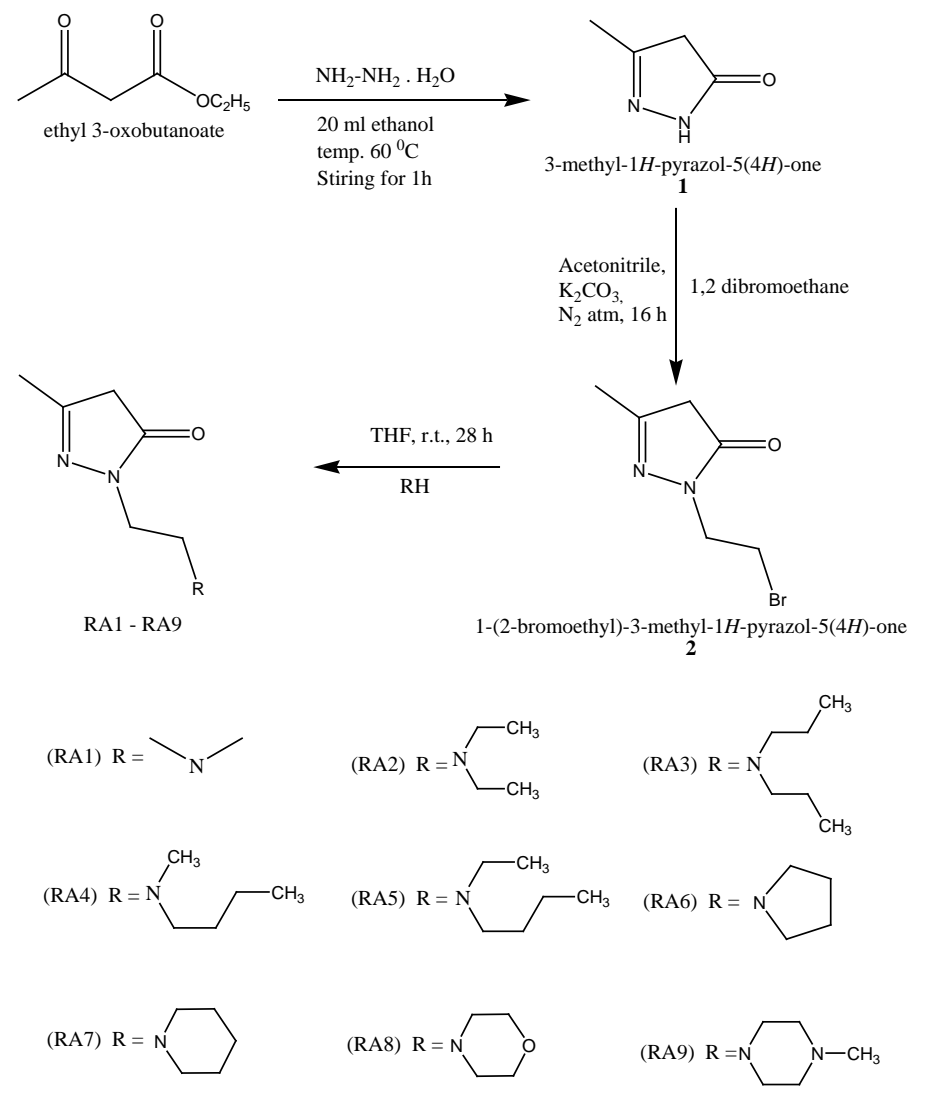

Scheme 1. Synthesis of compounds RA1-RA9. 


\section{Experimental}

Analytical TLC was performed on Silica gel F254 plates (Merck) with visualization by UV light. All the products obtained were purified by column chromatography using silica gel (100-200 mesh). Hexane was used as a co-eluent. ${ }^{1} \mathrm{H}$ NMR was recorded in Brucker $400 \mathrm{MHz}$ spectrometer. LC-MS was used for the mass spectral analysis. IR spectra were recorded on a FT-IR spectrometer using $\mathrm{KBr}$ pellets. Elemental analysis was carried out in CHN analyzer EA-1112, Thermo Finnigan. All spectral studies were carried out at elemental analysis laboratory in the School of Chemistry, University of Hyderabad, India. Melting points were determined in open capillaries on a Thermonik melting point apparatus, Mumbai, India and are uncorrected.

Synthesis of 3-methyl-1H-pyrazol-5(4H)-one (1): This compound was synthesized by methods reported earlier [20]. Ethyl acetoacetate ( 0.1 mole) was taken in conical flask and hydrazine hydrate $(0.2$ mole) in ethanol $(20 \mathrm{ml})$ was added drop wise to it with stirring. The temperature rose during this addition and it was maintained at $60{ }^{\circ} \mathrm{C}$ when a crystalline solid was formed. The reaction-mixture was further stirred for $1 \mathrm{~h}$ at room temperature, then cooled in an ice bath to complete the crystallization. Separated solid was washed with ice cold ethanol.

3-methyl-1H-pyrazol-5(4H)-one (1): Yield 82\%; mp 222-225 ${ }^{\circ} \mathrm{C}$; IR (KBr) $v \mathrm{~cm}^{-1}$ : 2997, 1651, 1551, 1502, 1453. ${ }^{1} \mathrm{H}-\mathrm{NMR}$ (DMSO-d6) $\delta$ : 10.50 (s, -NH, 2H), 5.20 (s, -CH, 1H), 2.07 (s, $\left.-\mathrm{CH}_{3}, 3 \mathrm{H}\right)$. MS m/z: $99(\mathrm{M}+1)$. Anal. Calcd for $\mathrm{C}_{4} \mathrm{H}_{6} \mathrm{~N}_{2} \mathrm{O}$ : C, 48.97; H, 6.16; N, 28.56. Found: C, 48.91; H, 6.84; N, 28.43.

Synthesis of 1-(2-bromoethyl)-3-methyl-1H-pyrazol-5(4H)-one (2): 3-methyl-1Hpyrazol-5(4H)-one (1) $(0.1 \mathrm{~mol})$ in $20 \mathrm{~mL}$ acetonitrile was added with $4 \mathrm{gm}$ of $\mathrm{K}_{2} \mathrm{CO}_{3}$. The mixture was stirred for 10 minutes under nitrogen atmosphere. 1,2-dibromo ethane ( $0.015 \mathrm{~mol})$ was added to this mixture which was stirred at room temperature for $16 \mathrm{~h}$ under nitrogen atmosphere. The completion of the reaction was monitored on silica gel 60 F254 precoated TLC plates (Merck) with visualization by UV light. After completion of reaction acetonitrile was removed under reduced pressure. The precipitate was collected; water and ethyl acetate (1:1) $50 \mathrm{~mL}$ were added to solid product. By using separating funnel organic layer was collected. Ethyl acetate was removed under reduced pressure and product collected. Further desired product was obtained by column chromatography and characterized by spectral studies.

1-(2-bromoethyl)-3-methyl-1H-pyrazol-5(4H)-one (2): Yield 53\%; mp 87-89 ${ }^{\circ} \mathrm{C}$; IR (KBr) $v \mathrm{~cm}^{-1}:$ 2997, 1651, 1551, 1501, 1218, 661. ${ }^{1} \mathrm{H}-\mathrm{NMR}$ (DMSO-d6) $\delta: 9.08$ (s, -NH, $1 \mathrm{H}), 5.50$ (s, - $\mathrm{CH}, 1 \mathrm{H}), 4.42\left(\mathrm{t},-\mathrm{CH}_{2}, 2 \mathrm{H}\right), 3.63\left(\mathrm{t},-\mathrm{CH}_{2}, 2 \mathrm{H}\right), 2.23\left(\mathrm{~s},-\mathrm{CH}_{3}, 3 \mathrm{H}\right) . \mathrm{MS} \mathrm{m} / \mathrm{z}$ : 207, 125. Anal. Calcd for $\mathrm{C}_{6} \mathrm{H}_{9} \mathrm{BrN}_{2} \mathrm{O}$ : C, 35.14; H, 4.42; N, 13.66. Found: C, 35.21; H, 4.28; N, 13.32. 
Synthesis of 1-(2-(dimethylamino)ethyl)-3-methyl-1H-pyrazol-5(4H)-one (RA1): 1-(2bromoethyl)3-methyl-1H-pyrazol-5(4H)-one (2) of $0.002 \mathrm{~mol}$ and $\mathrm{N}, \mathrm{N}$ dimethylamine 0.004 mol were mixed in a THF, and stirred at room temperature for $28 \mathrm{~h}$. THF was removed under reduced pressure. The product was collected and the desired product was isolated by column chromatography. The product was then characterized by spectral analysis. The compounds RA2-RA10 were obtained in a similar manner.

1-(2-(dimethylamino)ethyl)-3-methyl-1H-pyrazol-5(4H)-one (RA1): Yield 65\%; mp 103-105 ${ }^{\circ} \mathrm{C}$; IR (KBr) $v \mathrm{~cm}^{-1}:$ 2995, 1691, 1512, 1501, 1251. ${ }^{1} \mathrm{H}-\mathrm{NMR}$ (DMSO-d6) $\delta$ : 9.08 (s, -NH, 1H), 5.50 (s, -CH, 1H), 4.42 (t, - $\left.-\mathrm{CH}_{2}, 2 \mathrm{H}\right), 3.63$ (t, $\left.-\mathrm{CH}_{2}, 2 \mathrm{H}\right), 2.23\left(\mathrm{~s}, \mathrm{CH}_{3}\right.$, $3 \mathrm{H}), 2.35$ (s, $\left.\mathrm{CH}_{3}, 6 \mathrm{H}\right)$. MS m/z: $170(\mathrm{M}+1)$. Anal. Calcd for $\mathrm{C}_{8} \mathrm{H}_{15} \mathrm{~N}_{3} \mathrm{O}: \mathrm{C}, 56.78 ; \mathrm{H}, 8.93$; N, 24.83. Found: C, 56.54; H, 8.80; N, 24.33.

1-(2-(diethylamino)ethyl)-3-methyl-1H-pyrazol-5(4H)-one (RA2): Yield 62\%; mp 132$134{ }^{\circ} \mathrm{C}$; IR (KBr) $v \mathrm{~cm}^{-1}$ : 2999, 1694, 1523, 1501, 1254. ${ }^{1} \mathrm{H}-\mathrm{NMR}$ (DMSO-d6) $\delta: 9.08$ (s, -NH, 1H), 5.50 (s, -CH, 1H), $4.42\left(\mathrm{t},-\mathrm{CH}_{2}, 2 \mathrm{H}\right), 3.63\left(\mathrm{t},-\mathrm{CH}_{2}, 2 \mathrm{H}\right), 3.53\left(\mathrm{~s},-\mathrm{CH}_{3}, 6 \mathrm{H}\right)$, 3.13 (s, - $\left.-\mathrm{CH}_{3}, 3 \mathrm{H}\right), 2.45$ (m, - $\left.\mathrm{CH}_{2}, 2 \mathrm{H}\right), 2.39$ (m, - $\left.\mathrm{CH}_{2}, 2 \mathrm{H}\right) . \mathrm{MS}$ m/z: $198(\mathrm{M}+1)$. Anal. Calcd for $\mathrm{C}_{10} \mathrm{H}_{19} \mathrm{~N}_{3} \mathrm{O}$ : C, 60.88; H, 9.71; N, 21.30. Found: C, 60.44; H, 9.78; N, 21.13.

1-(2-(diisopropylamino)ethyl)-3-methyl-1H-pyrazol-5(4H)-one (RA3): Yield 69\%; mp 100-103 ${ }^{\circ} \mathrm{C}$; IR (KBr) $v \mathrm{~cm}^{-1}$ : 2997, 1697, 1514, 1258. ${ }^{1} \mathrm{H}-\mathrm{NMR}$ (DMSO-d6) $\delta: 9.08$ (s, $\mathrm{NH}, 1 \mathrm{H}), 5.50(\mathrm{~s},-\mathrm{CH}, 1 \mathrm{H}), 4.42\left(\mathrm{t},-\mathrm{CH}_{2}, 2 \mathrm{H}\right), 3.63\left(\mathrm{t},-\mathrm{CH}_{2}, 2 \mathrm{H}\right), 3.34\left(\mathrm{~d},-\mathrm{CH}_{3}, 12 \mathrm{H}\right)$, $2.88\left(\mathrm{~m},-\mathrm{CH}_{2}, 2 \mathrm{H}\right), 2.23$ (s, $\left.-\mathrm{CH}_{3}, 3 \mathrm{H}\right)$. MS m/z: $226(\mathrm{M}+1)$. Anal. Calcd for $\mathrm{C}_{12} \mathrm{H}_{23} \mathrm{~N}_{3} \mathrm{O}$ : C, 63.96; H, 10.29; N, 18.65. Found: C, 63.14; H, 10.87; N, 18.23.

1-(2-(butyl(methyl)amino)ethyl)-3-methyl-1H-pyrazol-5(4H)-one (RA4): Yield 72\%; mp 110-112 ${ }^{\circ} \mathrm{C}$; IR (KBr) $v \mathrm{~cm}^{-1}$ : 2998, 1692, 1523, 1509, 1260. ${ }^{1} \mathrm{H}-\mathrm{NMR}$ (DMSO-d6) $\delta$ : 9.08 (s, -NH, 1H), 5.50 (s, -CH, 1H), 4.42 (t, - $\left.\mathrm{CH}_{2}, 2 \mathrm{H}\right), 3.92\left(\mathrm{t},-\mathrm{CH}_{3}, 3 \mathrm{H}\right), 3.63\left(\mathrm{t},-\mathrm{CH}_{2}\right.$, $2 \mathrm{H}), 2.83\left(\mathrm{t},-\mathrm{CH}_{2}, 1 \mathrm{H}\right), 2.40\left(\mathrm{~m},-\mathrm{CH}_{2}, 1 \mathrm{H}\right), 2.23\left(\mathrm{~s},-\mathrm{CH}_{3}, 3 \mathrm{H}\right), 2.31\left(\mathrm{~m},-\mathrm{CH}_{2}, 1 \mathrm{H}\right), 2.12$ (s, $\left.-\mathrm{CH}_{3}, 1 \mathrm{H}\right)$. MS m/z: $212(\mathrm{M}+1)$. Anal. Calcd for $\mathrm{C}_{11} \mathrm{H}_{21} \mathrm{~N}_{3} \mathrm{O}$ : C, 62.52; H, 10.02; N, 19.89. Found: C, 62.42; H, 10.07; N, 19.20.

1-(2-(butyl(ethyl)amino)ethyl)-3-methyl-1H-pyrazol-5(4H)-one (RA5): Yield 65\%; mp 123-125 ${ }^{\circ} \mathrm{C}$; IR (KBr) $v \mathrm{~cm}^{-1}:$ 2987, 1691, 1501, 1268. ${ }^{1} \mathrm{H}-\mathrm{NMR}$ (DMSO-d6) $\delta: 9.08$ (s, $\mathrm{NH}, 1 \mathrm{H}), 5.50$ (s, -CH, 1H), 4.42 (t, - $\left.\mathrm{CH}_{2}, 2 \mathrm{H}\right), 3.82\left(\mathrm{t},-\mathrm{CH}_{3}, 6 \mathrm{H}\right), 3.63$ (t, - $\left.\mathrm{CH}_{2}, 2 \mathrm{H}\right), 2.83$ (t, $\left.-\mathrm{CH}_{2}, 1 \mathrm{H}\right), 2.40\left(\mathrm{~m},-\mathrm{CH}_{2}, 1 \mathrm{H}\right), 2.31\left(\mathrm{~m},-\mathrm{CH}_{2}, 1 \mathrm{H}\right), 2.23\left(\mathrm{~s},-\mathrm{CH}_{3}, 3 \mathrm{H}\right), 2.12\left(\mathrm{~s},-\mathrm{CH}_{3}\right.$, 1H). MS m/z: $226(\mathrm{M}+1)$. Anal. Calcd for $\mathrm{C}_{12} \mathrm{H}_{23} \mathrm{~N}_{3} \mathrm{O}$ : C, 63.96; H, 10.29; N, 18.65. Found: C, 63.23; H, 10.17; N, 18.29.

3-methyl-1-(2-(pyrrolidin-1-yl)ethyl)-1H-pyrazol-5(4H)-one (RA6): Yield 59\%; mp 118-119 ${ }^{\circ} \mathrm{C}$; IR (KBr) $v \mathrm{~cm}^{-1}:$ 2995, 1701, 1516, 1508, 1251. ${ }^{1} \mathrm{H}-\mathrm{NMR}$ (DMSO-d6) $\delta$ : 9.08 (s, -NH, 1H), 5.50 (s, -CH, 1H), 4.42 (t, - $\left.\mathrm{CH}_{2}, 2 \mathrm{H}\right), 3.63\left(\mathrm{t},-\mathrm{CH}_{2}, 2 \mathrm{H}\right), 2.41\left(\mathrm{t},-\mathrm{CH}_{2}\right.$, 
2H), $2.23\left(\mathrm{~s},-\mathrm{CH}_{3}, 3 \mathrm{H}\right), 2.12\left(\mathrm{~m},-\mathrm{CH}_{2}, 2 \mathrm{H}\right) . \mathrm{MS} \mathrm{m} / \mathrm{z}: 196(\mathrm{M}+1)$. Anal. Calcd for $\mathrm{C}_{10} \mathrm{H}_{17} \mathrm{~N}_{3} \mathrm{O}$ : C, 61.51; H, 8.78; N, 21.52. Found: C, 61.31; H, 8.43; N, 21.14.

3-methyl-1-(2-(piperidin-1-yl)ethyl)-1H-pyrazol-5(4H)-one (RA7): Yield 69\%; mp 129-130 ${ }^{\circ} \mathrm{C}$; IR (KBr) $v \mathrm{~cm}^{-1}:$ 2999, 1704, 1519, 1507, 1258. ${ }^{1} \mathrm{H}-\mathrm{NMR}$ (DMSO-d6) $\delta$ : 9.08 (s, -NH, 1H), 5.50 (s, -CH, 1H), $4.42\left(\mathrm{t},-\mathrm{CH}_{2}, 2 \mathrm{H}\right), 3.63\left(\mathrm{t},-\mathrm{CH}_{2}, 2 \mathrm{H}\right), 2.43\left(\mathrm{t},-\mathrm{CH}_{2}\right.$, $2 \mathrm{H}), 2.23\left(\mathrm{~s},-\mathrm{CH}_{3}, 3 \mathrm{H}\right), 2.14\left(\mathrm{~m},-\mathrm{CH}_{2}, 2 \mathrm{H}\right) . \mathrm{MS} \mathrm{m} / \mathrm{z}$ : $210(\mathrm{M}+1)$. Anal. Calcd for $\mathrm{C}_{11} \mathrm{H}_{19} \mathrm{~N}_{3} \mathrm{O}$ : C, 63.13; H, 9.15; N, 20.08. Found: C, 63.36; H, 9.32; N, 20.46.

3-methyl-1-(2-morpholinoethyl)-1H-pyrazol-5(4H)-one (RA8): Yield 65\%; mp 111$112{ }^{\circ} \mathrm{C}$; IR (KBr) $v \mathrm{~cm}^{-1}:$ 2988, 1700, 1517, 1509, 1261. ${ }^{1} \mathrm{H}-\mathrm{NMR}$ (DMSO-d6) $\delta: 9.08$ (s, -NH, $1 \mathrm{H}), 5.50$ (s, - $\mathrm{CH}, 1 \mathrm{H}), 4.42\left(\mathrm{t},-\mathrm{CH}_{2}, 2 \mathrm{H}\right), 3.63\left(\mathrm{t},-\mathrm{CH}_{2}, 2 \mathrm{H}\right), 2.23\left(\mathrm{~s},-\mathrm{CH}_{3}, 3 \mathrm{H}\right)$, $2.63\left(-\mathrm{CH}_{2}, 2 \mathrm{H}\right), 4.10\left(\mathrm{t},-\mathrm{CH}_{2}, 2 \mathrm{H}\right) . \mathrm{MS} \mathrm{m} / \mathrm{z}: 212(\mathrm{M}+1)$. Anal. Calcd for $\mathrm{C}_{10} \mathrm{H}_{17} \mathrm{~N}_{3} \mathrm{O}: \mathrm{C}$, 56.85; H, 8.11; N, 19.89. Found: C, 56.63; H, 8.12; N, 19.34.

3-methyl-1-(2-(4-methylpiperazin-1-yl)ethyl)-1H-pyrazol-5(4H)-one (RA9): Yield 68\%; mp 134-135 ${ }^{\circ} \mathrm{C}$; IR (KBr) $v \mathrm{~cm}^{-1}$ : 2995, 1709, 1515, 1505, 1268. ${ }^{1} \mathrm{H}-\mathrm{NMR}$ (DMSOd6) $\delta: 9.08$ (s, -NH, 1H), $5.50(\mathrm{~s},-\mathrm{CH}, 1 \mathrm{H}), 4.42\left(\mathrm{t},-\mathrm{CH}_{2}, 2 \mathrm{H}\right), 3.63\left(\mathrm{t},-\mathrm{CH}_{2}, 2 \mathrm{H}\right), 3.14$ (s, $\left.-\mathrm{CH}_{3}, 3 \mathrm{H}\right), 2.43\left(\mathrm{t},-\mathrm{CH}_{2}, 2 \mathrm{H}\right), 2.23\left(\mathrm{~s},-\mathrm{CH}_{3}, 3 \mathrm{H}\right) . \mathrm{MS} \mathrm{m} / \mathrm{z}: 225(\mathrm{M}+1)$. Anal. Calcd for $\mathrm{C}_{11} \mathrm{H}_{20} \mathrm{~N}_{4} \mathrm{O}$ : C, 58.90; H, 8.99; N, 24.98. Found: C, 58.36; H, 8.23; N, 24.14.

\section{Biological Activities}

\subsection{Antitumor Activity}

In vitro anticancer activity of compounds RA1-RA9 against EAC cells was determined by trypan blue exclusion method [16]. The EAC cells were collected, counted and adjusted to 106 cells/mL with normal saline. The drug dilutions were made with phosphate buffer saline (PBS) and were further adjusted to concentrations ranging from 125-1000 $\mu \mathrm{g} / \mathrm{mL}$. The drug dilutions were then added to the EAC cells and incubated at $37^{\circ} \mathrm{C}$ for $3 \mathrm{~h}$. At the end of $3 \mathrm{~h}$, the cell viability was determined and percentage cytotoxicity was calculated. The percentage cytotoxicity was calculated using the formula:

Percentage cytotoxicity $=100-T_{\mathrm{C}}-D_{\mathrm{C}} / T_{\mathrm{C}} \times 100$

where, $T_{\mathrm{C}}=$ total EAC cells and $D_{\mathrm{c}}=$ dead EAC cells [17].

Compounds RA1-RA9 with significant in vitro anticancer activity were further selected for screening in vivo anticancer activity by determining different parameters like body weight analysis, mean survival time (MST) and percentage increase in life span (\% ILS) [18]. The EAC cells containing 106 cells/ $0.1 \mathrm{~mL}$ of phosphate buffer saline were injected into the peritoneal cavity of all the animals (six swiss albino mice in each group). Treatment with test compounds (90 mg/kg body weight) and the standard 5-flurouracil 
(520 $\mu \mathrm{g} / \mathrm{kg}$ body weight) was started $24 \mathrm{~h}$ after inoculation of cancer cells, once daily as a single dose in $0.3 \%$ CMC suspension by intraperitoneal route for 10 days. All the mice were weighed daily up to 11 days. The decrease in body weight and MST (Mean Survival Time) of the test and standard group animals were compared with control group. Results are shown in Table 1. Percentage decrease in the body weight was determined by using the formula, percentage decrease in body weight $=\left(G_{\mathrm{c}}-G_{\mathrm{t}}\right) / G_{\mathrm{c}} \times 100$, where $G_{\mathrm{c}}=$ gain in body weight of control group and $G_{\mathrm{t}}=$ gain in body weight of treated group. Percentage increase in life span was calculated by the formula, \% ILS = (MST of treated group - MST of control group) $/$ MST of control group $\times 100$. Student t-test was performed to ascertain the significance of the exhibited activity.

Table 1. In-vitro and in-vivo anticancer activity of compounds (RA1-RA9).

\begin{tabular}{|c|c|c|c|c|c|c|c|c|}
\hline \multirow[t]{2}{*}{ Compounds } & \multicolumn{4}{|c|}{$\begin{array}{l}\text { In-vitro anticancer activity \% cyto- } \\
\text { toxicity of drugs at various conc. on } \\
\text { EAC cells }(\mu \mathrm{g} / \mathrm{ml})\end{array}$} & \multicolumn{4}{|c|}{ In-vivo anticancer activity } \\
\hline & 900 & 600 & 300 & 150 & $\begin{array}{l}\text { Gain in body } \\
\text { weight }\end{array}$ & $\begin{array}{l}\text { \% decrease } \\
\text { in body wt. }\end{array}$ & $\mathrm{MST}^{\mathrm{a})} \pm$ S.E. ${ }^{\mathrm{b})}$ & $\%$ ILS $^{\mathrm{c}}$ \\
\hline Control & - & - & - & - & $5.01 \pm 0.02$ & - & $14.10 \pm 0.23$ & - \\
\hline RA1 & 87.0 & 79.0 & 56.1 & 49.2 & $0.88 \pm 0.028$ & 82.43 & $34.50 \pm 0.19$ & $144.68^{* *}$ \\
\hline RA2 & 82.7 & 72.8 & 41.9 & 39.7 & $0.94 \pm 0.023$ & 81.23 & $30.85 \pm 0.24$ & $112.76^{* *}$ \\
\hline RA3 & 79.1 & 70.8 & 40.1 & 37.3 & $1.03 \pm 0.064$ & 79.44 & $29.18 \pm 0.25$ & $106.95^{* *}$ \\
\hline RA4 & 86.1 & 74.4 & 53.0 & 42.1 & $0.84 \pm 0.023$ & 83.23 & $35.80 \pm 0.18$ & $153.90^{* *}$ \\
\hline RA5 & 81.5 & 72.6 & 41.9 & 39.8 & $0.95 \pm 0.14$ & 81.03 & $29.86 \pm 0.29$ & $111.77^{* *}$ \\
\hline RA6 & 26.1 & 22.4 & 15.0 & 11.8 & $2.30 \pm 0.32$ & 54.09 & $17.53 \pm 0.24$ & $24.32^{*}$ \\
\hline RA7 & 16.7 & 13.6 & 11.3 & 9.1 & $4.15 \pm 0.11$ & 17.16 & $15.70 \pm 0.05$ & $11.34^{* *}$ \\
\hline RA8 & 19.1 & 18.7 & 12.8 & 10.1 & $3.17 \pm 0.14$ & 36.72 & $16.49 \pm 0.38$ & $16.95^{*}$ \\
\hline RA9 & 89.2 & 73.5 & 53.0 & 47.1 & $0.83 \pm 0.010$ & 99.40 & $37.50 \pm 0.19$ & $165.95^{* *}$ \\
\hline 5-flurouracil & 83.1 & 72.3 & 43.2 & 40.1 & $0.91 \pm 0.013$ & 81.83 & $32.15 \pm 0.25$ & $128.01^{* *}$ \\
\hline
\end{tabular}

${ }^{\text {a) }}$ MST = mean survival time, ${ }^{\text {b) }}$ S.E. = standard error, ${ }^{\text {c) }} \%$ ILS = percentage increase in life span, ${ }^{* *} P<0.01{ }^{*} P<0.05$.

\section{Hematological analysis [17]}

In order to detect the influence of RA1-RA9 on hematological status of EAC-bearing mice, a comparison was made among four groups $(n=6)$ of mice on the $13^{\text {th }}$ day after tumor inoculation. Group I comprised of normal mice, group II comprised of EAC bearing mice, group III comprising EAC bearing mice treated with (RA1-RA9) (90 $\mathrm{mg} / \mathrm{kg} /$ day for 10 days), and group IV having EAC bearing mice treated with 5-flurouracil ( $520 \mu \mathrm{g} / \mathrm{kg}$ for 10 days). Blood was drawn from each mouse by the retro orbital plexus method and the white blood cell count (WBC), red blood cell count (RBC), hemoglobin and percentage differential count were determined. The results are given in Table 2. 
Table 2. Determination of hematological parameters of (RA1-RA9).

\begin{tabular}{ccccccc}
\hline Compounds & Hb (g \%) & $\begin{array}{c}\text { RBC } \\
\left.\text { (million } / \mathrm{mm}^{3}\right)\end{array}$ & $\begin{array}{c}\text { WBC } \\
\left(10^{3}\right. \\
\left.\text { cells } / \mathrm{mm}^{3}\right)\end{array}$ & Lymphocytes & Neutrophils & Monocytes \\
\cline { 5 - 7 } & & & & & & \\
\hline RA1 & $13.5 \pm 0.07$ & $4.2 \pm 0.06$ & $13.6 \pm 0.08$ & $74.3 \pm 0.19$ & $31.1 \pm 0.11$ & $1 \pm 0$ \\
RA2 & $12.7 \pm 0.07$ & $3.8 \pm 0.08$ & $14.5 \pm 0.12$ & $72.2 \pm 0.19$ & $28.1 \pm 0.11$ & $1 \pm 0$ \\
RA3 & $12.5 \pm 0.10$ & $3.7 \pm 0.06$ & $14.8 \pm 0.08$ & $71.3 \pm 0.18$ & $27.1 \pm 0.16$ & $2 \pm 0$ \\
RA4 & $13.4 \pm 0.41$ & $4.1 \pm 0.10$ & $13.6 \pm 0.07$ & $74.5 \pm 0.11$ & $30.0 \pm 0.07$ & $1 \pm 0$ \\
RA5 & $12.8 \pm 0.09$ & $3.8 \pm 0.06$ & $15.3 \pm 0.12$ & $73.2 \pm 0.38$ & $28.1 \pm 0.12$ & $1 \pm 0$ \\
RA6 & $7.0 \pm 0.13$ & $2.4 \pm 0.10$ & $19.3 \pm 0.08$ & $29.1 \pm 0.12$ & $71.1 \pm 0.19$ & $2 \pm 0$ \\
RA7 & $6.2 \pm 0.07$ & $2.8 \pm 0.08$ & $18.0 \pm 0.19$ & $31.3 \pm 0.19$ & $69.2 \pm 0.28$ & $2 \pm 0$ \\
RA8 & $6.9 \pm 0.10$ & $3.0 \pm 0.08$ & $21.6 \pm 0.73$ & $25.4 \pm 0.30$ & $73.5 \pm 0.44$ & $2 \pm 0$ \\
RA9 & $13.6 \pm 0.05$ & $4.2 \pm 0.08$ & $13.3 \pm 0.08$ & $74.5 \pm 0.26$ & $30.0 \pm 0.05$ & $1 \pm 0$ \\
5-flurouracil & $13.3 \pm 0.10$ & $4.1 \pm 0.09$ & $13.8 \pm 0.12$ & $73.2 \pm 0.28$ & $31.0 \pm 0.08$ & $1 \pm 0$ \\
I & $14.2 \pm 0.31$ & $4.5 \pm 0.06$ & $7.0 \pm 0.08$ & $75.1 \pm 0.11$ & $29.1 \pm 0.12$ & $1 \pm 0$ \\
II & $7.2 \pm 0.12$ & $2.2 \pm 0.09$ & $22.1 \pm 0.11$ & $23.5 \pm 0.29$ & $74.1 \pm 0.10$ & $2 \pm 0$ \\
\hline
\end{tabular}

$\mathrm{I}$ = group comprising normal mice, $\mathrm{II}$ = group having EAC bearing mice.

\subsection{Antibacterial and antifungal activities}

Applying the agar plate diffusion technique [19] all of the newly synthesized compounds were screened in vitro for antibacterial activity against Escherichia coli (E. coli), Pseudomonas aeruginosa (Gram-negative), Staphylococcus aureus, Bacillus subtilis (Gram-positive) at $20 \mu \mathrm{g} / \mathrm{ml}, 30 \mu \mathrm{g} / \mathrm{ml}, 40 \mu \mathrm{g} / \mathrm{ml}$ concentrations, respectively. Under identical conditions, the positive control antibiotics Amoxicillin at $40 \mu \mathrm{g} / \mathrm{ml}$ showed zone of inhibition 26-24 mm and 22-25 mm for Gram-negative and Gram-positive organism respectively. Similarly, the antifungal screening of the compounds was carried out against fungi C. albicans and A. niger by using fluconazole $(40 \mathrm{mg} / \mathrm{ml})$ as the positive control, which showed $27 \mathrm{~mm}$ and $28 \mathrm{~mm}$, respectively, as the zone of inhibition.

\section{Results and Discussion}

In vivo antitumor screening reveals that some of the tested compounds are promising candidates having good activity against EAC cells. Compounds RA1, RA4, and RA9 exhibit highest antitumor activity than standard antitumor agent. Compounds RA2, RA3, RA5 exhibit nearly same antitumor activity comparable to standard. Compounds RA6, RA7, RA8 are inactive against EAC cells. Antimicrobial study reveals that compounds RA1, RA4, RA6 and RA9 exhibit good antibacterial and antifungal activities (Tables 3 and 4). Compound RA9 exhibited the highest degree of antimicrobial activity than standard drugs. Rest of the compounds does not show any significant antimicrobial activity as compared with standard agent. 
Table 3. Antibacterial Activity of Compounds ${ }^{\mathrm{a}}$ (RA1-RA9).

\begin{tabular}{ccccccccccccc}
\hline & \multicolumn{3}{c}{ Escherichia coli } & \multicolumn{3}{c}{ Pseudomonas aeruginosa } & \multicolumn{3}{c}{ Bacillus subtilis } & \multicolumn{3}{c}{ Staphylococcus aureus } \\
\cline { 2 - 11 } Comp. & $\begin{array}{c}20 \\
(\mu \mathrm{g} / \mathrm{ml})\end{array}$ & $\begin{array}{c}30 \\
(\mu \mathrm{g} / \mathrm{ml})\end{array}$ & $\begin{array}{c}40 \\
(\mu \mathrm{g} / \mathrm{ml})\end{array}$ & $\begin{array}{c}20 \\
(\mu \mathrm{g} / \mathrm{ml})\end{array}$ & $\begin{array}{c}30 \\
(\mu \mathrm{g} / \mathrm{ml})\end{array}$ & $\begin{array}{c}40 \\
(\mu \mathrm{g} / \mathrm{ml})\end{array}$ & $\begin{array}{c}20 \\
(\mu \mathrm{g} / \mathrm{ml})\end{array}$ & $\begin{array}{c}30 \\
(\mu \mathrm{g} / \mathrm{ml})\end{array}$ & $\begin{array}{c}40 \\
(\mu \mathrm{g} / \mathrm{ml})\end{array}$ & $\begin{array}{c}20 \\
(\mu \mathrm{g} / \mathrm{ml})\end{array}$ & $\begin{array}{c}30 \\
(\mu \mathrm{g} / \mathrm{ml})\end{array}$ & $\begin{array}{c}40 \\
(\mu \mathrm{g} / \mathrm{ml})\end{array}$ \\
\hline RA1 & 11 & 18 & 23 & 13 & 18 & 23 & 13 & 20 & 24 & 12 & 19 & 24 \\
RA2 & - & - & - & 4 & 6 & 9 & 3 & 7 & 11 & 5 & - & 8 \\
RA3 & 3 & 7 & 10 & 2 & 5 & 9 & 4 & 7 & 10 & 4 & 7 & 11 \\
RA4 & 10 & 15 & 22 & 12 & 20 & 23 & 11 & 17 & 23 & 12 & 18 & 22 \\
RA5 & 4 & 7 & 10 & 3 & 7 & 11 & 3 & 6 & 12 & 4 & 6 & 12 \\
RA6 & 10 & 16 & 22 & 11 & 14 & 21 & 10 & 17 & 22 & 12 & 18 & 22 \\
RA7 & 5 & 9 & 12 & - & - & - & 4 & 8 & 13 & 4 & 7 & 9 \\
RA8 & 6 & 10 & 15 & 5 & 7 & 11 & 6 & 9 & 13 & 6 & 8 & 10 \\
RA9 & 13 & 23 & 28 & 14 & 22 & 26 & 14 & 21 & 28 & 14 & 22 & 26 \\
Amoxicillin & 12 & 20 & 26 & - & - & 24 & 14 & 22 & 27 & 12 & - & 25 \\
\hline
\end{tabular}

${ }^{\mathrm{a}}=$ Zone of inhibition in millimeter.

Table 4. Antifungal Activity of Compounds ${ }^{\mathrm{a}}$ (RA1-RA9).

\begin{tabular}{ccccccc}
\hline & \multicolumn{3}{c}{ Candida albicans } & \multicolumn{3}{c}{ Aspergillus niger } \\
\cline { 2 - 7 } Compounds & $\begin{array}{c}20 \\
(\mu / \mathrm{ml})\end{array}$ & $\begin{array}{c}30 \\
(\mu / \mathrm{ml})\end{array}$ & $\begin{array}{c}40 \\
(\mu / \mathrm{ml})\end{array}$ & $\begin{array}{c}20 \\
(\mu / \mathrm{ml})\end{array}$ & $\begin{array}{c}30 \\
(\mu / \mathrm{ml})\end{array}$ & $\begin{array}{c}40 \\
(\mu / \mathrm{ml})\end{array}$ \\
\cline { 2 - 7 } RA1 & 12 & 18 & 24 & 11 & 20 & 25 \\
RA2 & 5 & 7 & 14 & 3 & - & - \\
RA4 & 3 & 6 & 10 & 2 & 4 & 5 \\
RA5 & 11 & 19 & 24 & 14 & 21 & 27 \\
RA6 & 13 & 7 & 10 & 5 & 9 & 17 \\
RA7 & 5 & 6 & 12 & 3 & 7 & 11 \\
RA8 & 4 & 6 & 9 & 1 & 4 & 6 \\
RA9 & 15 & 22 & 27 & 13 & 22 & 29 \\
Amoxicillin & 14 & 23 & 27 & - & - & 28 \\
\hline a Zone of inhibition in millimeter. & & & &
\end{tabular}

Structure activity studies of the title compounds for antitumor activity reveal the importance of $\mathrm{N}-1$ position of pyrazolone ring as it should contain $\mathrm{N}$-methyl substitution followed by ethyl linkage as RA1 (144.68), RA4 (153.90), RA9 (165.95) increases the percentage increases in life span (\%ILS) as compared to compound RA6 (24.32), RA7 
(11.34), RA8 (16.95) having heterocyclic substitution at N-1 position followed by ethyl linkage.

Hematological parameters of EAC bearing mice on day 13 showed significant changes when compared to normal mice (Table 2). The total WBC count was found to increase with a reduction in the hemoglobin content of RBC. The differential count of WBC showed that the percentage of neutrophils increased while that of lymphocytes decreased. At the same time treatment with compounds RA1, RA4, and RA9 could change these altered parameters to near normal.

\section{Acknowledgements}

We gratefully acknowledge the UGC Networking Centre, School of Chemistry, University of Hyderabad, for providing laboratory facilities for synthesis and Spectral Analysis. IISc (Bangalore) for the Spectral analysis. Also we are thankful to the Divakar Goli, Principal, Acharya \& B.M. Reddy College of Pharmacy for providing laboratory facilities.

\section{References}

1. C. Daniele, D. L. Alessandro, R. Marco, B. Beatrice, M. Fabrizio, and M. Matteo, Bioorg. Med. Chem. 16, 8587 (2008). http://dx.doi.org/10.1016/j.bmc.2008.08.016

2. R. V. Ragavan, V. Vijayakumar, and N. S. Kumari, Eur. J. Med. Chem. 44, 3852 (2009). http://dx.doi.org/10.1016/j.ejmech.2009.04.010

3. M. F. Brana, A. Gradillas, A. G. Ovalles, B. Lopez, N. Acero, and F. Llinares, Bioorg. Med. Chem. 14, 9 (2006). http://dx.doi.org/10.1016/j.bmc.2005.09.059

4. K. R. Kim, J. L. Kwon, J. S. Kim, N. Zaesung, H. R. Kim, and H. G. Cheon, Eur. J. Pharmacol. 528, 37 (2005). http://dx.doi.org/10.1016/j.ejphar.2005.10.027

5. X. Fan, X. Zhang, L. Zhou, K. A. Keith, E. R. Kernb, and P. F. Torrencea, Bioorg. Med. Chem. Lett. 16, 3224 (2006). http://dx.doi.org/10.1016/j.bmcl.2006.03.043

6. V. C. Filho, R. Correa, Z. Vaz, J. B. Calixto, R. J. Nunes, T. R. Pinheiro, A. D. Andricopulo, and R. A.Yunes, Farmaco. 53, 55 (1998). doi:10.1016/S0014-827X(97)00006-2

7. S. M. Sondhi, Sharma, R. P. Verma, N. Singhal, R. Shukla, R. Raghubir, and M. P. Dubey, Synthesis.878 (1999). http://dx.doi.org/10.1055/s-1999-3472

8. M. M. F. Ismail, Y. A. Ammar, H. S. A. El-Zahaby, S. I. Eisa, and S. E. Barakat, Arch. Pharm. Life. Sci. 340, 476 (2007).

9. A. M. El-Hawash, A. M. B. El-Sayed, and I. M. El-Ashmawey, Eur. J. Med. Chem. 41, 155 (2006). http://dx.doi.org/10.1016/j.ejmech.2005.09.006

10. F. R. Souza, V. T. Souza, V. Ratzlaff, L. P. Borges, M. R. Oliveira, H. G. Bonacorso, N. Zanatta, M. A. P. Martins, and C. F. Mello, Eur. J. Pharmacol. 451, 141 (2002). http://dx.doi.org/10.1016/S0014-2999(02)02225-2

11. A. Mohd and S. Kumar, Indian J. Chem. 44 B, 2532 (2005).

12. M. P. Clark, S. K. Laughlin, M. J. Laufersweiler, R. G. Bookland, T. A. Brugel, A. Golebiowski, and M. P. Sabat, J. Med. Chem. 47, 2724 (2004). http://dx.doi.org/10.1021/jm049968m

13. S. Kuppusamy, S. Gnanamani, P. S. Nagarajan, M. Seeralan, T. P. Paramasivan, and R. Melani, Bioorg. Med. Chem. Lett. 19, 4501 (2009). http://dx.doi.org/10.1016/j.bmcl.2009.02.113

14. P. Camilleri, D. P. Astles, M. W. Kerr and J. E. Spencer, J. Agric. Food. Chem. 38, 1601 (1990). http://dx.doi.org/10.1021/jf00097a036

15. A. Kimata, H. Nakagawa, R. Ohyama, T. Fukuuchi, S. Ohta, T. Suzuki, and N. Miyata, J. Med. Chem. 50, 5053 (2007). http://dx.doi.org/10.1021/jm070688r 


\section{2}

16. V. Murugan, M. Kulkarni, R. M. Anand, E. P. Kumar, B. Suresh, and V. M. Reddy, Asian J. Chem. 18, 900 (2006).

17. N. M. Raghavendra, P. M. Gurubasavarajaswamy, K. S. Nagaranavile, and T. Parameshwaran, Arch. Pharm. Res. 32 (3), 431 (2009). http://dx.doi.org/10.1007/s12272-009-1317-8

18. J. A. Khanam, S. P. Bag, B. Sur, and P. Sur, Ind. J. Pharmacol. 29, 157 (1997).

19. V. Mathew, J. Keshavayya, V. P. Vaidya, and D. Giles, Eur. J. Med. Chem. 42, 823 (2007). http://dx.doi.org/10.1016/j.ejmech.2006.12.010

20. C. Sharma, B. Thadhaney, G. Pemawat, and G. L. Talesara, Indian J. Chem. 47 B, 1892 (2008). 